\title{
A COSMO-ONTOLOGICAL ARGUMENT FOR THE EXISTENCE OF A FIRST CAUSE - PERHAPS GOD
}

\author{
UWE MEIXNER
}

University of Augsburg

\begin{abstract}
The paper presents a new version of the "Cosmological Argument" - considered to be an ontological argument, since it exclusively uses ontological concepts and principles. It employs famous results of modern physics, and distinguishes between event-causation and agent-causation. Due to these features, the argument manages to avoid the objection of infinite regress. It remains true, however, that the conclusion of the argument (just like the conclusion of Thomas Aquinas's causal argument) is too unspecific to be unambiguously considered an argument for the existence of God.
\end{abstract}

I should say a few words about why I presented at the Bydgoszcz conference about "ontological proofs" ideas that are relevant for what has come to be known as "cosmological proofs". First, aside from the special meaning Kant has given the designations "ontological proof" and "cosmological proof", a so-called cosmological proof is at least as much an ontological argument as a so-called ontological proof. Both sorts of argument aim to establish the existence of God, and existence is, of course, a central - perhaps the central - ontological concept. Second, one might even say that so-called cosmological arguments, if they exclusively use ontological concepts, like the argument I am going to present here, are more ontological in kind than many so-called ontological arguments. After all, Anselm's original version of what Kant was the first to call an ontological argument, and many later versions, involve an epistemic and an axiological concept: the being such that no greater being can be conceived. Third, in my view, the interest of so-called ontological proofs is mainly logical, not theological, and not metaphysical. - Well, may this suffice as my excuse for what follows. 
There is a kind of causation where the cause is sufficient for the realization of the effect (that is, for the realization of what is caused), the effect being some event: an entity involving a finite temporal region, particulars, and properties (relational and non-relational ones) had by these particulars within that temporal region:

(A1) Effects (i.e. what is caused) are always events.

In sufficient causation, the cause determines the coming-about of the effect-event; the cause does not make the effect-event merely probable, or more probable than it would be without the cause, and the cause is not merely an indispensable factor for the coming-about of the effect-event. In sufficient event-causation, the coming-about of an event determines the coming-about of the effect-event. In sufficient agent-causation, simply the agent determines the coming-about of the effect-event.

In what follows, the phrase "a cause of" will always mean the same as "a sufficient cause of", and "to cause" will always mean the same as "to be a sufficient cause of". And these phrases will always be understood to exclude self-causation: Nothing is a cause of itself. (One may wish to count this as axiom (A0).) The definition of the concept that is central to this paper is this:

(D) A first cause is a cause without a cause, in other, fully explicit words: a first sufficient cause is a sufficient cause (of some event), but a sufficient cause that itself has no sufficient cause.

It is easily seen:

(T1) If agents are not events, then every agent that is a cause is a first cause. Suppose we have an agent that is a cause, i.e. that causes some event. If agents are not events, then that agent is not an event, hence it has no cause (for otherwise it would be an effect, and therefore an event, since effects are always events according to (A1)).

Now indeed:

(A2) Agents are not events, but substances. And therefore:

(T2) Every agent that is a cause is a first cause.

Hence:

(T3) If there are agents that are causes, then there are first causes. But are there agents that are causes? That there are such items is doubted by many, even denied. Doubtless, however, there are events that are 
causes. And if one could find an event that is a cause, but has no cause, then this causal event - though not a causal agent - would also serve as a perfect first cause. But are there events that are causes without having a cause? We do not have purely scientific evidence for the existence of such events. What we do have purely scientific evidence for is merely this:

(A3) Some physical events are causes, but there is no physical event that causes them.

Now, at this point, there is a crucial decision to be made in causationtheory. It is not an empirical, it is not a scientific, it is not a conceptual decision; it is a genuinely metaphysical decision. A choice is to be made between two very plausible metaphysical principles. One of these two principles is known as the principle of (sufficient) causation:

(A4.1) Every event has a cause.

The other principle is one of the principles known as principles of physical causal closure:

(A4.2) Every physical event that has a cause is caused by a physical event. One cannot adopt both principles - because, unfortunately, their conjunction is not compatible with (A3). On the other hand, each of the two principles under consideration has so many credentials on its side that it seems rationally inappropriate to reject both. Let's see what the consequences would be if one accepted the one, or the other.

(A4.2) is the modernization of a materialist, or physicalist, principle that emerged as a metaphysical side-effect of the rise of modern physics. This original principle is the following:

$\left(\right.$ A4. $\left.2^{\star}\right)$ Every physical event is caused by a physical event.

This latter principle was adopted by all who, inspired by impressive scientific progress, considered a purely immanent world-view - a worldview without transcendence - to be the only rational world-view. The insertion of "that has a cause" after "Every physical event" - which is of no detriment to the original metaphysical motivation - became necessary due to the developments in physics in the $20^{\text {th }}$ century; these developments make modern physics entail the falsity of (A4.2*). (A4.2), however, is left quite untouched by them.

Now, obviously, the conjunction of (A3) and (A4.2) logically entails that there are physical events that are causes, but have no cause. Thus, if we add (A4.2) to our list of axiomatic principles (and not (A4.1)), then 
the existence of first causes is established. There are, then, first causes in the form of physical events that are causes without having a cause.

In contrast, it is a straightforward logical consequence of (A4.1) that no event is a first cause. For if an event is a cause, (A4.1) requires that it also be caused, that is: have a cause. (A4.1) is a principle that throughout the roughly 2500 years of the history of philosophy was almost universally accepted by the philosophers as an absolute requirement of rationality, comparable in this to a law of logic. And when (A4.2*) became prominent in the philosophical consciousness (roughly 300 years ago), it peacefully coexisted there with (A4.1); indeed, one could regard (A4.2*) as a mere specialization of (A4.1), as merely spelling out what it is that (A4.1) means for physical events. All that changed in the $20^{\text {th }}$ century with the establishment of quantum physics and empirical cosmology, and hence of the scientific fact that is stated by (A3). (A3) refutes $\left(\mathrm{A} 4.2^{*}\right)$, and it also refutes the conjunction of the logically weaker (A4.2) with (A4.1). But (A3) neither refutes (A4.2) taken by itself, nor does it refute (A4.1) taken by itself. If, tentatively, we add (A4.1) to our list of axiomatic principles (and not (A4.2)), continuing thereby the very long and almost univocal philosophical tradition in favour of (A4.1), we get an interesting result: There are physical events that have a cause, though they are not caused by any physical event.

Given (A3), one cannot adopt (A4.1) and (A4.2) together, and it does not seem rationally right to reject both. A choice, therefore, has to be made between these two principles. There is no argument that would rationally force one to choose (A4.1) rather than (A4.2). But since (A4.1) involves much less of a metaphysical commitment than (A4.2); since, in other words, the rational appeal of (A4.1) is more general than that of (A4.2), and less dependent on the rationality of a specific metaphysical motivation, I herewith adopt (A4.1) as axiomatic, and as a consequence change its label from "(A4.1)" to simply "(A4)":

(A4) Every event has a cause.

And with both (A4) and (A3) as axioms, we now have as a theorem:

(T4) There are physical events that have a cause, though they are not caused by any physical event.

But, of course, with (A4) as an axiom, there is no chance that an event is a first cause; if there are first causes, then they must be something other than events. In fact, they must be agents, since the following is true:

(A5) Every cause is an agent or an event. 
(A5) makes it possible to derive:

(T5) Every first cause is an agent.

Assume that $\mathrm{X}$ is a first cause, and assume also that $\mathrm{X}$ is an event. But then, according to (A4), $\mathrm{X}$ has a cause, and is, therefore, not a first cause - contrary to the first assumption. Therefore (holding on to that assumption): $\mathrm{X}$ is not an event, and therefore: $\mathrm{X}$ is an agent (because of (A5), and because $\mathrm{X}$ is, qua first cause, also a cause).

I am well aware that some philosophers have proposed facts, or even properties, as causes. But causes must be causally effective, and a property, taken by itself, is not causally effective; a property is only then causally effective - in an analogical way - if it is had, exemplified, instantiated by an object in such a way that the resulting fact is causally effective. But a fact, in its turn, is only then causally effective - in a derivative, secondary way - if it is replaceable in this role by a causal event. Causation by facts, in other words, is reducible to causation by events. There is, therefore, no substantial reason to reject (A5).

(T4) gives rise to the following considerations: Suppose $\mathrm{E}^{\star}$ is one of the physical events that - according to (T4) - have a cause, though there is no physical event that causes them. Thus:

(a) $\mathrm{E}^{\star}$ is a physical event.

(b) $\mathrm{E}^{\star}$ has a cause.

(c) There is no physical event that causes $\mathrm{E}^{\star}$.

Hence, by making use of (A5), we have:

(d) $\mathrm{E}^{\star}$ has a cause that is a nonphysical event or an agent.

Assume now the following additional axiomatic principles:

(A6) Every event that is caused by an event is also caused by an event that is not caused by any event.

(A7) For all $x, y$ and $z$ : if $x$ causes $y$, and $y$ causes $z$, then $x$ causes $z$. (A7) expresses the transitivity of (sufficient!) causation - one of the most uncontroversial principles in causation theory. (A6), in turn, is the Limit Principle for the Causation by Events. This, to some, may seem like a very problematic principle; it actually is no such thing. Suppose (A6) is wrong, and $\mathrm{E}$ is an event that is caused by an event, but there is no event that causes $\mathrm{E}$ and is not caused by any event. It is easily seen (employing (A7)) that a consequence of this supposition is the following: all causal chains of events that end with $E$ are infinite or incomplete. 
Suppose $\mathrm{C}$ is a causal chain of events which ends with $\mathrm{E}$ and which is neither infinite nor incomplete. (Note that for a normal conception of a causal chain i.e. for the exclusion of its being a loop - the truth of (A0) is necessary.) Since C is not an infinite causal chain of events, there is a first event in C, call it " $\mathrm{E}_{1}$ ". Since $\mathrm{C}$ is a complete causal chain of events, there is no event that causes $\mathrm{E}_{1}$. Given the transitivity of causation (i.e. the truth of (A7)) and given that $C$ ends with $E_{1}, E_{1}$ causes $\mathrm{E}$. Thus there is an event (namely, $\mathrm{E}_{1}$ ) that causes $\mathrm{E}$ and is not caused by any event - contradicting the supposition which introduced $\mathrm{E}$ in the first place.

Is this consequence of negating (A6) for some event $\mathrm{E}$ - the consequence that all E-ending causal chains of events are infinite or incomplete - more reasonable a priori than (A6)? I don't think so. Is this consequence more reasonable on empirical grounds than (A6)? I don't think it is, certainly not given today's physics.

Using the two principles last introduced, we obtain from (d):

(e) $\mathrm{E}^{\star}$ is caused by an agent.

The first alternative in (d) leads to the result that $\mathrm{E}^{*}$ is caused by an agent, just as does (trivially) the second alternative in (d). Suppose the first alternative in (d) is true: $\mathrm{E}^{\star}$ is caused by a nonphysical event. With (A6) we obtain: $\mathrm{E}^{\star}$ is caused by an event $\mathrm{E}^{\prime}$ that is not caused by any event. ${ }^{1}$ But according to (A4): E' has a cause, $\mathrm{G}$. Since $\mathrm{E}^{\prime}$ is not caused by any event, $\mathrm{G}$ must be an agent (according to (A5)). Since G causes E' and E' causes $E^{\star}$, it follows according to (A7): $G$ causes $\mathrm{E}^{\star}$. Therefore: $\mathrm{E}^{\star}$ is caused by an agent.

Consequently we get on the basis of (T2):

(f) There is an agent that is a first cause.

And this result - since, ultimately, it is a logical consequence purely of the axiomatic principles (A0) to (A7) - is a theorem: a statement logically proven on the basis of those axioms:

(T6) There is an agent that is a first cause.

This result chimes perfectly with the penultimate result of what has traditionally, since Kant, been called "the Cosmological Argument for the Existence of God". But although Thomas Aquinas nonchalantly concludes from the penultimate conclusion of the Cosmological Argument - that there is a first cause (which Thomas certainly thought to be an agent) - its

${ }^{1}$ Note that E' must be a nonphysical event. Otherwise, E' would be a physical event that causes $\mathrm{E}^{*}$ - contradicting $(\mathrm{c})$. 
ultimate conclusion: that there is $\mathrm{God},{ }^{2}$ it must nonetheless be emphasized that this is a very problematic last step. Nothing in Thomas Aquinas's argument, and nothing in the modernization of it here presented: nothing in (T6) and the axiomatic principles on which (T6) is based, justifies the conclusion that this agent which is a first cause is God or even $a$ god.

But, of course, the modernized Cosmological Argument I have presented can be strengthened. In order to see just at what point it can be strengthened, consider first the compact presentation of the argument as it is now:

(A0) Nothing is a cause of itself.

(A1) Effects are always events.

(A2) Agents are not events, but substances.

(A3) Some physical events are causes, but there is no physical event that causes them.

(A4) Every event has a cause.

(A5) Every cause is an agent or an event.

(A6) Every event that is caused by an event is also caused by an event that is not caused by any event.

(A7) For all $x, y$ and $z$ : if $x$ causes $y$, and $y$ causes $z$, then $x$ causes $z$. [together logically entail among other things]

(T6) There is an agent that is a first cause.

Replace now (A3) by $\left(\mathrm{A}^{\star}{ }^{*}\right)$ (leaving the other axioms - or premises just as they are):

$\left(A 3^{*}\right)$ The Big Bang is a physical event that is a cause, but there is no physical event that causes it.

The specific principle $\left(\mathrm{A} 3^{*}\right)$ is just as true from the point of view of modern physics as the unspecific (A3). With it and the rest of the axioms as premises, one can logically deduce:

$\left(T 6^{*}\right)$ There is an agent that is a first cause of the Big Bang.

From $\left(\mathrm{A} 3^{*}\right)$ and $(\mathrm{A} 4)$ we get: $\mathrm{BB}$ is a physical event that has a cause, but there is no physical event that causes BB. Let A be a cause of BB. According to (A5), A is an agent or an event.

2 "Ergo est necesse ponere aliquam causam efficientem primam: quam omnes Deum nominant" (S. Th. I, qu. 2, a. 3; see the conclusion of the secunda via). 
In case $\mathrm{A}$ is an agent, $\mathrm{A}$ is not an event (according to (A2)), and therefore $\mathrm{A}$ is not an effect (according to (A1)), i.e. A is not caused, in other words: A has no cause. But A causes BB. Thus: there is an agent (namely, A) that is a first cause of BB.

In case $\mathrm{A}$ is an event, it follows on the basis of (A6) that $\mathrm{BB}$ is also caused by an event that is not caused by any event. Let E' be such an event. It follows on the basis of (A4) that there is a cause of E', and on the basis of (A5) it follows that that cause (any such cause) can only be an agent (it cannot be an event, since $E^{\prime}$ is not caused by any event). Let $A^{\prime}$ be such an agent. A' causes $E^{\prime}$, and $E^{\prime}$ causes BB, and therefore (according to (A7)): $\mathrm{A}^{\prime}$ causes BB. Moreover, since $\mathrm{A}^{\prime}$ is an agent, it is not an event (see (A2)), and therefore not an effect (see (A1)), i.e. $A^{\prime}$ is not caused, in other words: $A^{\prime}$ has no cause. Thus we have again: there is an agent (namely, $\mathrm{A}^{\prime}$ ) that is a first cause of BB.

An agent that is a first cause of the Big Bang - that is: of the initial event of the Physical World - does seem to be godlike. By excluding the causation of the same event (any event) by several agents - which is a plausible theoretical step - we can even obtain that there is one and only one agent that is a first cause of the Big Bang. Moreover, also in line with traditional theism, the agent that causes the initiation of space-time-energy-matter can hardly be denied to be nonphysical. However, nothing so far shows that this agent is different from, say, what Schopenhauer called "the Will", different from a blind, irrational, and basically evil - but transcendent - source of the Universe. That the First Cause of the beginning of the Universe is different from such a being is a matter of faith. But, note, it is also a matter of faith that God Himself is different from such a being.

Neither the axioms nor the theorems in this paper seem to me utterly speculative, epistemologically irresponsible, or irrational. I certainly believe that they provide food for serious thought. Yet there are, of course, objections. I will consider three of them (which actually came up when I presented the paper at the Bydgoszcz conference).

Objection 1 (against $\left(\mathrm{A}^{*}\right)$ ): The Big Bang does not exist, because the Big Bang, if it is anything, is the total physical event which occurs at the first moment of time, and there is no first moment of time (as Stephen Hawking has famously held). Response: Even if there is no first moment of time, it does not follow that there is no initial physical event. Note that events, though they are required to be temporally finite according to the notion of event here employed (see the beginning of this paper), are not required by that notion to have a first or a last moment. An initial physical event is a physical event whose temporal region is the initial 
interval of time - and that interval may be an interval that is open on one side, even on both sides. The Big Bang, then, is the total physical event whose temporal region is the initial interval of time. One might further object that there is not only no first moment of time, but also no initial interval of time. But, by the lights of modern physics (which may be wrong of course, but there is no guide in these matters that is known to be better), the initial interval of time is simply the first interval of time whose duration is the Planck-time (that is, $10^{-43} \mathrm{sec}$ ). There certainly is such an interval of time (even if there is no first moment of time), and the corresponding event - the Big Bang - is, as far as we know, correctly described by $\left(\mathrm{A} 3^{*}\right)$.

Objection 2 (against (A3) being the entire scientifically warranted truth): There is purely scientific evidence not only for (A3) but also for the existence of physical events that are causes without having a cause. For it is a scientific principle that if a physical event is not caused by any physical event then it is not caused by anything. Response: The objection relies on (A4.2) - which is a principle of causal closure - being a scientific principle. No doubt, many scientists employ that principle; but that, by itself, does not make it a scientific principle. ${ }^{3}$ In fact, (A4.2) is not a scientific, but a metaphysical principle - just like (A4.1), the principle of causation. It is a metaphysical principle because logical, mathematical, empirical, and methodological-esthetical considerations alone are not sufficient for warranting its assumption.

Objection 3: The notion of agent causation, which is necessary for obtaining (T6) and $\left(\mathrm{T}^{*}\right)$, is an irremediably unclear notion. When, for example, does agent causation happen? Response: This is a stock objection, the merits of which are doubtful. For one thing, the notion of event causation is not so clear either (and yet we continue to use it, and could not well do without it). For another thing, I have offered a detailed analysis of agent causation in my books Ereignis und Substanz and The Two Sides of Being, in the former regarding both creatural and divine agency, in the latter regarding only creatural agency. A comprehensive theory of causation, both of event causation and agent causation, can be found in my book Theorie der Kausalität, also containing extensive

\footnotetext{
${ }^{3}$ Many scientists in the past have made successful use - within the very context of their scientific endeavours - of the hypothesis that God exists and has created the Universe (for example, Johannes Kepler in his arduous search for the laws of planetary motion). But that, of course, does not imply that the existence of God is a scientific principle.
} 
discussions of the literature. Some of the main results of Theorie der Kausalität are presented in my paper "Causation in a New Old Key". The emergence of creatural agent causation in the course of natural history is defended in several of my papers, for example, "The Emergence of Rational Souls" and "New Perspectives for a Dualistic Conception of Mental Causation".

And when does agent causation "happen"? Instances of agent causation do not happen, since they - in contrast to the effects involved in them - are not events (and only events can happen). But if one absolutely wishes to assign a time to an instance of agent causation, then it is simply the time of the effect that is involved in it.

\section{BIBLIOGRAPHY}

Meixner, U. Ereignis und Substanz. Die Metaphysik von Realität und Realisation (Paderborn: Schöningh, 1997)

Meixner, U. Theorie der Kausalität. Ein Leitfaden zum Kausalbegriff in zwei Teilen (Paderborn: Mentis, 2001)

Meixner, U. The Two Sides of Being. A Reassessment of Psycho-Physical Dualism (Paderborn: Mentis, 2004)

Meixner, U. 'Causation in a New Old Key', Studia Logica, 76 (2004), 343-383

Meixner, U. 'New Perspectives for a Dualistic Conception of Mental Causation', Journal of Consciousness Studies, 15, No. 1 (2008), 17-38

Meixner, U. 'The Emergence of Rational Souls', in Emergence in Science and Philosophy, edited by A. Corradini and T. O'Connor (New York/London: Routledge (Taylor \& Francis), 2010), pp. 163-179. 